\title{
Semi-analytical homologous solutions of the gravo-magnetic contraction
}

\author{
P. Hennebelle $e^{1,2, \star}$ \\ ${ }^{1}$ Laboratoire de radioastronomie millimétrique, UMR 8112 du CNRS, École Normale Supérieure et Observatoire de Paris, \\ 24 rue Lhomond, 75231 Paris cedex 05, France \\ 2 Department of Physics and Astronomy, Cardiff University, PO Box 913, 5 The Parade, Cardiff CF24 3YB, Wales, UK
}

Received 12 November 2001 / Accepted 17 July 2003

\begin{abstract}
We propose an extension of the semi-analytical solutions derived by Lin et al. (1965) describing the two-dimensional homologous collapse of a self-gravitating rotating cloud having uniform density and spheroidal shape, which includes magnetic field (with important restrictions) and thermal pressure. The evolution of the cloud is reduced to three time-dependent ordinary equations allowing one to conduct a quick and preliminary investigation of the cloud dynamics during the precollapse phase, for a wide range of parameters. We apply our model to the collapse of a rotating and magnetized oblate and prolate isothermal core. Hydrodynamical numerical simulations are performed and comparison with the semi-analytical solutions is discussed. Under the assumption that all cores are similar, an apparent cloud axis ratio distribution is calculated from the sequence of successive evolutionary states for each of a large set of initial conditions. The comparison with the observational distribution of the starless dense cores belonging to the catalog of Jijina et al. (1999) shows a good agreement for the rotating and initially prolate cores (aspect ratio $\simeq 0.5$ ) permeated by an helical magnetic field $\left(\simeq 17-20 \mu \mathrm{G}\right.$ for a density of $\simeq 10^{4} \mathrm{~cm}^{-3}$ ).
\end{abstract}

Key words. accretion, accretion disks - gravitation - hydrodynamics - magnetohydrodynamics (MHD) - ISM: clouds

\section{Introduction}

Stars form through the gravitational collapse of dense cores located in the molecular clouds. These condensations are complex and involve gravity, hydrodynamics, magnetic and thermal processes.

The core dynamics has been investigated numerically by several authors. Although the numerical approach undoubtedly allows a deep understanding of the cloud dynamics, it does not replace the analytical studies, first because analytical work gives more explicit descriptions and second because the complexity of numerical methods keeps increasing, making a full exploration of the space parameters and their use by non numerical experts difficult.

Analytically, most of the efforts have been dedicated to the description of the isothermal gravitational collapse (Larson 1969; Penston 1969a, 1969b; Hunter 1977; Shu 1977; Whitworth \& Summers 1985; Bouquet et al. 1985). These solutions that assume self-similarity are very useful to get a physical hint of how the collapse proceeds and to give strong benchmarks to the numerical studies. However in spite of its importance, very few analytical tools are available to describe the dynamics of the precollapsing or starless phase. The present paper is an attempt to fill this gap.

\footnotetext{
^ e-mail: patrick.hennebelle@ens.fr
}

In this paper, we first present new solutions of the gravomagnetic condensation. They are an extension of solutions obtained by Lin et al. (1965) for the gravitational part and simultaneously by Aburihan et al. (2001) and by the author (Hennebelle 2001) for the MHD part. We then use these solutions to study the phenomenology of the collapse of oblate and prolate, isothermal, magnetized and non magnetized clouds and compare the result with the evolution obtained with numerical simulations. The solutions allow to calculate the cloud shape evolution. With the assumption that all cores are similar, distributions of apparent axis ratio are predicted. We then compare these distributions to the apparent axis ratio distribution of the starless dense cores belonging to the catalog of Jijina et al. (1999).

Indeed the geometry of the dense cores is an important issue since it depends on the physical processes that drive their evolution. Previous investigations by Myers et al. (1991) and Ryden (1996) conclude that the observational data are more consistent with axisymmetric prolate cores than with axisymmetric oblate cores. The same conclusion is reached by the recent study of Curry (2002) with a different sample of cores and by Hartmann (2002) who shows that the major axis of the cores in the Taurus cloud is preferentially aligned with the filament in which they are embedded. Two recent studies (Jones et al. 2001 and Goodwin et al. 2002) analyse the 
observational apparent axis ratio distribution derived from the catalog of Jijina et al. (1999) and conclude that the core apparent axis ratio distribution is compatible with triaxal cores but being more nearly oblate than prolate. From a theoretical point of view, the equilibrium solutions of a rotating and magnetized cloud (Mouschovias 1976; Tomisaka et al. 1988) present an oblate shape except if there is a substantial toroidal magnetic component (Tomisaka 1991) or if the structure of the poloidal magnetic field is such that it compresses the cloud (Stahler \& Curry 2001). Curry (2000) also shows that two-dimensional solutions of the isothermal Lane-Emden equation present a prolate configurations. On the other hand, recent studies show (Nakamura et al. 1993; Fiege \& Pudritz 2000a,b) that prolate cores can be formed from a magnetized filament (with poloidal and toroidal field) through a combination of the gravitational and the magnetic sausage instabilities. Fiege \& Pudritz (2000c) also demonstrate that the axis ratio of the prolate magnetized cores is compatible with the observational apparent axis ratio distribution.

In the second section of the paper, we derive the solutions and reduce the self-gravitating MHD equations to three time dependent ordinary equations. We discuss their mathematical and physical properties, their potential and intrinsic weaknesses. In the third section, we solve these ordinary equations numerically and describe the phenomenology of the collapse of an isothermal dense core. We also compare the semi-analytical solutions with the results of the hydrodynamical numerical simulations of an isothermal dense core. The fourth section is devoted to the comparison between the theoretical and observational apparent axis ratio distributions. A discussion and summary are given in the fifth part.

\section{Homologous solutions of the gravo-magnetic condensation}

In this section we look for self-similar solutions of Eqs. (1)-(10) and reduce this system into a system of three ordinary differential equations of the time.

In most of the classic studies of self-similar solutions (Larson 1969; Penston 1969a,b; Hunter 1977; Shu 1977; Whitworth \& Summers 1985; Bouquet et al. 1985), a special and simple time dependence of the fields is assumed leading to ordinary equations of the self-similar variable. These solutions are indeed related to the invariance of the self-gravitating hydrodynamical equations through the dilatation groups (see e.g. Olver 1986 and Ferrara \& Shchekinov 1996 for an introduction to the application of Lie groups to differential equations).

In this paper, on the contrary, we first assume a special and simple spatial dependence and put all the complexity in the time dependence of the fields. This technique has been successfully applied by Lin et al. (1965) to predict the eccentricity evolution of a cold cloud and by Hennebelle \& Pérault (2000) to study the thermo-magnetic condensation.

\subsection{The equations}

We consider the perfect MHD equations of self-gravitating gas with polytropic equation of state and assume an axisymmetric geometry. With the usual notations, we have in cylindrical coordinates:

$$
\begin{aligned}
& \partial_{t} \rho+\frac{1}{\varpi} \partial_{\varpi}\left(\varpi V_{\varpi} \rho\right)+\partial_{z}\left(V_{z} \rho\right)=0, \\
& \rho\left(\partial_{t} V_{\varpi}+V_{\varpi} \partial_{\varpi} V_{\varpi}+V_{z} \partial_{z} V_{\varpi}-\frac{V_{\theta}^{2}}{\varpi}\right)=-\partial_{\varpi} P \\
& \quad+\rho \partial_{\varpi} \Phi-\frac{1}{\mu_{0} \varpi} B_{\theta} \partial_{\varpi}\left(\varpi B_{\theta}\right)+\frac{1}{\mu_{0}} B_{z}\left(\partial_{z} B_{\varpi}-\partial_{\varpi} B_{z}\right),
\end{aligned}
$$

$$
\begin{array}{r}
\rho\left(\partial_{t} V_{\theta}+V_{\varpi} \partial_{\varpi} V_{\theta}+V_{z} \partial_{z} V_{\theta}+\frac{V_{\theta} V_{\varpi}}{\varpi}\right)= \\
\frac{1}{\mu_{0} \varpi} B_{\varpi} \partial_{\varpi}\left(\varpi B_{\theta}\right)+\frac{1}{\mu_{0}} B_{z} \partial_{z} B_{\theta},
\end{array}
$$

$\rho\left(\partial_{t} V_{z}+V_{\varpi} \partial_{\varpi} V_{z}+V_{z} \partial_{z} V_{z}\right)=-\partial_{z} P$

$+\rho \partial_{z} \Phi-\frac{1}{\mu_{0}} B_{\theta} \partial_{z} B_{\theta}-\frac{1}{\mu_{0}} B_{\varpi}\left(\partial_{z} B_{\varpi}-\partial_{\varpi} B_{z}\right)$,

$\partial_{t}\left(P \rho^{-\gamma}\right)+V_{\varpi} \partial_{\varpi}\left(P \rho^{-\gamma}\right)+V_{z} \partial_{z}\left(P \rho^{-\gamma}\right)=0$,

$\frac{1}{\varpi} \partial_{\varpi}\left(\varpi \partial_{\varpi} \Phi\right)+\partial_{z^{2}}^{2} \Phi=-4 \pi G \rho$,

$\frac{1}{\varpi} \partial_{\varpi}\left(\varpi B_{\varpi}\right)+\partial_{z} B_{z}=0$

$\partial_{t} B_{\varpi}-\partial_{z}\left(V_{\varpi} B_{z}-V_{z} B_{\varpi}\right)=0$

$\partial_{t} B_{\theta}+\partial_{z}\left(V_{z} B_{\theta}-V_{\theta} B_{z}\right)-\partial_{\varpi}\left(V_{\theta} B_{\varpi}-V_{\varpi} B_{\theta}\right)=0$,

$\partial_{t} B_{z}+\frac{1}{\varpi} \partial_{\varpi}\left(\varpi\left(V_{\varpi} B_{z}-V_{z} B_{\varpi}\right)\right)=0$.

\subsection{Reduction to time-dependent ordinary differential equations}

Let us define the Jeans frequency:

$\Omega_{J}=\sqrt{4 \pi G \rho_{0}}$,

and

$t=\tau / \Omega_{J}$,

where $\rho_{0}$ is the gas density. We consider the following fields:

$$
\begin{aligned}
\rho(\tau, \varpi, z) & =\rho_{0} d(\tau) \\
P(\tau, \varpi, z) & =\rho_{0} \Omega_{J}^{2}\left(P_{c}(\tau)-p_{\varpi}(\tau) \varpi^{2}-p_{z}(\tau) z^{2}\right), \\
\Phi(\tau, \varpi, z) & =-\Omega_{J}^{2} d(\tau)\left(A_{\varpi}(\tau) \varpi^{2}+A_{z}(\tau) z^{2}\right), \\
V_{\varpi}(\tau, \varpi, z) & =\frac{\dot{a}(\tau)}{a(\tau)} \Omega_{J} \varpi, \\
V_{\theta}(\tau, \varpi, z) & =\dot{\theta}(\tau) \Omega_{J} \varpi, \\
V_{z}(\tau, \varpi, z) & =\frac{\dot{c}(\tau)}{c(\tau)} \Omega_{J} z \\
B_{\varpi}(\tau, \varpi, z) & =\sqrt{\mu_{0} \rho_{0}} \Omega_{J} h_{\varpi}(\tau) \varpi, \\
B_{\theta}(\tau, \varpi, z) & =\sqrt{\mu_{0} \rho_{0}} \Omega_{J} h_{\theta}(\tau) \varpi, \\
B_{z}(\tau, \varpi, z) & =B_{c}(\tau)+\sqrt{\mu_{0} \rho_{0}} \Omega_{J} h_{z}(\tau) z .
\end{aligned}
$$


where the dotted values denotes derivative against $\tau$ and where $d, P_{c}, p_{\varpi}, p_{z}, A_{\varpi}, A_{z}, a, c, \dot{\theta}, h_{\varpi}, h_{z}, h_{\theta}$, and $B_{c}$ are functions of $\tau$.

The definitions stated by Eqs. (13) lead with Eqs. (1)-(10) to the following ordinary differential equations:

Continuity:

$\dot{d}(\tau)+\left(2 \frac{\dot{a}(\tau)}{a(\tau)}+\frac{\dot{c}(\tau)}{c(\tau)}\right) d(\tau)=0$.

Energy conservation:

$\frac{\mathrm{d}}{\mathrm{d} \tau}\left(P_{0}(\tau) d^{-\gamma}(\tau)\right)=0$,

$\frac{\mathrm{d}}{\mathrm{d} \tau}\left(p_{\varpi}(\tau) d^{-\gamma}(\tau)\right)+2 \frac{\dot{a}(\tau)}{a(\tau)}\left(p_{\varpi}(\tau) d^{-\gamma}(\tau)\right)=0$,

$\frac{\mathrm{d}}{\mathrm{d} \tau}\left(p_{z}(\tau) d^{-\gamma}(\tau)\right)+2 \frac{\dot{c}(\tau)}{c(\tau)}\left(p_{z}(\tau) d^{-\gamma}(\tau)\right)=0$.

Poisson equation:

$4 A_{\varpi}(\tau)+2 A_{z}(\tau)=1$.

Momentum conservation:

$d(\tau)\left(\frac{\ddot{a}(\tau)}{a(\tau)}+\dot{\theta}^{2}(\tau)\right)=2 p_{\varpi}(\tau)-2 A_{\varpi}(\tau) d(\tau)^{2}-2 h_{\theta}(\tau)^{2}$,

$d(\tau)\left(\ddot{\theta}(\tau)+2 \frac{\dot{a}(\tau)}{a(\tau)} \dot{\theta}(\tau)\right)=2 h_{\varpi}(\tau) h_{\theta}(\tau)$,

$d(\tau) \frac{\ddot{c}(\tau)}{c(\tau)}=2 p_{z}(\tau)-2 A_{z}(\tau) d(\tau)^{2}$.

Nullity of magnetic divergence:

$2 h_{\varpi}(\tau)+h_{z}(\tau)=0$.

Induction equation:

$\dot{h}_{\varpi}(\tau)-\frac{\dot{a}(\tau)}{a(\tau)} h_{z}(\tau)+\frac{\dot{c}(\tau)}{c(\tau)} h_{\varpi}(\tau)=0$,

$\dot{h}_{\theta}(\tau)+\left(2 \frac{\dot{a}(\tau)}{a(\tau)}+\frac{\dot{c}(\tau)}{c(\tau)}\right) h_{\theta}(\tau)-\dot{\theta}(\tau)\left(2 h_{\varpi}(\tau)+h_{z}(\tau)\right)=0$,

$\dot{h}_{z}(\tau)+2 \frac{\dot{a}(\tau)}{a(\tau)} h_{z}(\tau)-2 \frac{\dot{c}(\tau)}{c(\tau)} h_{\varpi}(\tau)=0$,

$\dot{B}_{c}(\tau)+2 \frac{\dot{a}(\tau)}{a(\tau)} B_{c}(\tau)=0$.

Equations (14)-(17), (23)-(26) are straightforwardly integrated and lead to:

$d(\tau)=\frac{1}{a(\tau)^{2} c(\tau)}$,

$P_{c}(\tau)=P^{0} d(\tau)^{\gamma}$,

$p_{\varpi}(\tau)=p_{\varpi}^{0} \frac{d(\tau)^{\gamma}}{a(\tau)^{2}}$, $p_{z}(\tau)=p_{z}^{0} \frac{d(\tau)^{\gamma}}{c(\tau)^{2}}$

$h_{\varpi}(\tau)=\frac{h_{\varpi}^{0}}{a(\tau)^{2} c(\tau)}$

$h_{\theta}(\tau)=\frac{h_{\theta}^{0}}{a(\tau)^{2} c(\tau)}$,

$h_{z}(\tau)=-2 \frac{h_{\varpi}^{0}}{a(\tau)^{2} c(\tau)}$,

$B_{c}(\tau)=\frac{B^{0}}{a(\tau)^{2}}$,

where $P^{0}, p_{\varpi}^{0}, p_{z}^{0}, h_{\varpi}^{0}, h_{\theta}^{0}, B^{0}$ are real numbers.

Defining $W(\tau)=a(\tau)^{2} \dot{\theta}(\tau)$, the system of Eqs. (14)-(26) reduces after some algebra, to the three differential ordinary equations:

$\ddot{a}(\tau)=\frac{2 p_{\pi}^{0}}{a(\tau)^{2 \gamma-1} c(\tau)^{\gamma-1}}+\frac{W^{2}(\tau)}{a(\tau)^{3}}-\frac{2 A_{\varpi}(\tau)}{a(\tau) c(\tau)}-\frac{2\left(h_{\theta}^{0}\right)^{2}}{a(\tau) c(\tau)}$,

$\ddot{c}(\tau)=\frac{2 p_{z}^{0}}{a(\tau)^{2 \gamma-2} c(\tau)^{\gamma}}-\frac{2 A_{z}(\tau)}{a(\tau)^{2}}$,

$\dot{W}(\tau)=\frac{2 h_{\theta}^{0} h_{\tau}^{0}}{c(\tau)}$.

\subsection{Gravitational potential}

In the system of Eqs. (35)-(37), the gravitational force (i.e. $A_{\varpi}$ and $A_{z}$ ) is unknown and must be specified. Its value indeed depends on the boundary conditions. For simplicity, it can be assumed that $A_{\varpi}$ and $A_{z}$ remain constant along time, as it was the case in the studies carried out by Hennebelle (2001). However the problem of boundary conditions has not been addressed for these solutions. It is likely that because of boundary conditions, rotation and magnetic field will induce an evolution of the shape of the cloud (and consequently a variation of $A_{\varpi}$ and $A_{z}$ ) for most of these solutions (if cylindrical geometry is assumed consistent solutions can be obtained, see Hennebelle 2003).

More consistently, if one considers elliptical boundary conditions, i.e. a uniform axisymmetric spheroid, then one knows the gravitational potential as a function of the spheroid eccentricity $e$ (Mestel 1963; Lin et al. 1965; Chandrasekhar 1969; Binney \& Tremaine 1987). This allows to address properly the problem of boundary conditions and to obtain self-consistent solutions.

For an oblate spheroid, we have:

$$
\begin{aligned}
& A_{\varpi}(e)=\frac{1}{4} \frac{\sqrt{1-e^{2}}}{e^{2}}\left(\frac{\arcsin (e)}{e}-\sqrt{1-e^{2}}\right) \\
& A_{z}(e)=\frac{1}{2} \frac{\sqrt{1-e^{2}}}{e^{2}}\left(\frac{1}{\sqrt{1-e^{2}}}-\frac{\arcsin (e)}{e}\right),
\end{aligned}
$$


and consequently:

$$
\begin{aligned}
e \rightarrow 0 & \Rightarrow\left\{\begin{array}{l}
A_{\varpi} \rightarrow 1 / 6 \\
A_{z} \rightarrow 1 / 6
\end{array}\right. \\
e \rightarrow 1 & \Rightarrow\left\{\begin{array}{l}
A_{\varpi} \simeq(c / a)^{2} \rightarrow 0 \\
A_{z} \rightarrow 1 / 2
\end{array}\right.
\end{aligned}
$$

For a prolate spheroid, we have:

$$
\begin{aligned}
& A_{\varpi}(e)=\frac{1}{4} \frac{1-e^{2}}{e^{2}}\left(\frac{1}{1-e^{2}}-\frac{1}{2 e} \ln \left(\frac{1+e}{1-e}\right)\right), \\
& A_{z}(e)=\frac{1}{2} \frac{1-e^{2}}{e^{2}}\left(\frac{1}{2 e} \ln \left(\frac{1+e}{1-e}\right)-1\right) .
\end{aligned}
$$

and:

$$
\begin{aligned}
& e \rightarrow 0 \Rightarrow\left\{\begin{array}{l}
A_{\varpi} \rightarrow 1 / 6 \\
A_{z} \rightarrow 1 / 6
\end{array}\right. \\
& e \rightarrow 1 \Rightarrow\left\{\begin{array}{l}
A_{\varpi} \rightarrow 1 / 4 \\
A_{z} \simeq(a / c)^{2} \ln (a / c) \rightarrow 0
\end{array}\right.
\end{aligned}
$$

$l_{\varpi}$ and $l_{z}$ being the length of the radial and the polar axis respectively, the eccentricity is given by:

$$
e(\tau)=\sqrt{1-\left(\frac{l_{z}}{l_{\varpi}}\right)^{2}},
$$

for an oblate spheroid $\left(l_{\varpi}>l_{z}\right)$, and by:

$$
e(\tau)=\sqrt{1-\left(\frac{l_{\varpi}}{l_{z}}\right)^{2}},
$$

for a prolate spheroid $\left(l_{\varpi}<l_{z}\right)$.

The lengths of the axis evolve with time according to:

$$
l_{\varpi}(\tau)=l_{\varpi}(0) a(\tau), l_{z}(\tau)=l_{z}(0) c(\tau)
$$

\subsection{Physical interpretations}

The system of Eqs. (35)-(37) is of second order in $a$ and $c$ and of first order in $W$. It thus depends on 5 initial conditions, however one can assume without any restriction that $a(0)=$ 1 and $c(0)=1$. We thus have the 3 initial conditions, $\dot{a}(0)$, $\dot{c}(0)$, and $W(0)$ which respectively represent the initial radial velocity, axial velocity and rotation.

We also have the 5 parameters $P^{0}, \gamma, h_{\varpi}^{0}, h_{\theta}^{0}$ and $e(0)$ (as we will see with Eq. (49), $e(0), P^{0}, p_{\varpi}^{0}$ and $p_{z}^{0}$ are indeed related) which represent respectively the initial thermal pressure, the polytropic index, the initial magnetic poloidal field and magnetic toroidal field and the initial eccentricity.

The system of Eqs. (35)-(37) is much simpler than the system of Eqs. (1)-(10) and in spite of important physical restrictions, allows to study various non-linear aspects of the gravitational contraction. Before solving Eqs. (35)-(37) numerically, and in order to anticipate the numerical results, we discuss the physical meaning and mathematical behaviour of each of the terms in these three equations.

\subsubsection{The radial momentum conservation}

Equation (35) derives from the radial momentum equation, the first term of the right-hand side is the thermal pressure, the second is the centrifugal force, the third is the gravitational force, and the last is the toroidal magnetic pressure. The poloidal magnetic pressure does not appear since it cancels out (the poloidal magnetic field is force free) as it was already the case in the studies of Aburihan et al. (2001) and Hennebelle (2001).

Since the density is uniform, the thermal pressure gradient is due to a non uniform temperature field. Although such temperature gradients are expected if the gas is opaque to its radiation, in the case of dense cores that we will consider in the next sections the temperature should be nearly constant. Rigorously speaking, these temperature gradients should be seen as ersatz that allows to treat the thermal pressure semi-analytically.

- If the collapse is spherical $\left(p_{\varpi}^{0}=p_{z}^{0}, e(0)=0, W(0)=0\right.$, $\left.h_{\theta}^{0}=0\right), a=c$ and Eq. (35) can be integrated once leading in the case $\gamma \neq 1$ to:

$\frac{1}{2} \dot{a}^{2}=\frac{2}{3-3 \gamma} \frac{p_{\varpi}^{0}}{a^{3 \gamma-3}}+\frac{1}{3} \frac{1}{a}+K$,

where $K$ is a constant which depends on the initial conditions. If $\gamma \leq 4 / 3$ the collapse is possible, since the gravitational force dominates the pressure force in the limit $a \rightarrow 0$. The collapse is not possible if $\gamma>4 / 3$.

- If the cloud contracts radially, we can assume that $c$ remains constant during the final stage of the collapse. Remembering Eq. (41), Eq. (35) can be further integrated (provided $\gamma \neq 1)$ in the limit $a \ll c_{0}\left(c(\tau) \simeq c_{0}\right)$ :

$$
\begin{aligned}
\frac{1}{2} \dot{a}^{2} \simeq \frac{2}{2-2 \gamma} \frac{p_{\varpi}^{0}}{a^{2 \gamma-2} c_{0}^{\gamma-1}} & -\frac{2 W^{2}(0)}{a^{2}} \\
& -\left(\frac{1}{2 c_{0}}+\frac{2\left(h_{\theta}^{0}\right)^{2}}{c_{0}}\right) \ln (a)+K,
\end{aligned}
$$

where $K$ again is an integration constant. Therefore, thermal pressure cannot support the cloud if $\gamma \leq 1$ whereas if $\gamma>$ 1 , it prevents the collapse. The centrifugal force prevents the collapse and the Lorentz force due to the toroidal magnetic field adds up to gravity.

- If the collapse occurs along the polar axis only $(c \rightarrow 0)$, the pressure force is proportional to: $c^{1-\gamma}$, gravity is proportional to $c$ (remembering Eqs. (39), $A_{\varpi} \propto c^{2}$ ) and the toroidal force is proportional to $1 / c$. Consequently, the radial component of the gravitational force becomes small compared to the thermal pressure whereas on the contrary, the toroidal magnetic force dominates the thermal pressure.

\subsubsection{The axial momentum conservation}

Equation (36) derives from the axial momentum equation. It contains only the thermal pressure (first term of right-hand side) and gravity (second term) since the magnetic forces in our geometry are radial and azimuthal.

- If the cloud contracts radially $(a \rightarrow 0)$, then with Eqs. (41), we find that the pressure force is proportional to 
$a^{2-2 \gamma}$ and gravity to $\ln (a)$, consequently the thermal pressure dominates if $\gamma>1$.

- If the collapse occurs along the pole $(c \rightarrow 0)$, assuming that $a$ remains constant ( $a \simeq a_{0}$ ), we can integrate Eq. (36) once (assuming that $\gamma \neq 1$ ):

$\frac{1}{2} \dot{c}^{2} \simeq \frac{2}{1-\gamma} \frac{p_{\pi}^{0}}{a_{0}^{2 \gamma-2} c^{\gamma-1}}-\frac{1}{a_{0}^{2}} c+K$,

consequently, the collapse into an infinitely thin disk is not possible if $\gamma \geq 1$. As pointed out in the conclusion of Lin et al. (1965), at some point the cloud may rebound.

\subsubsection{The azimuthal momentum conservation}

Equation (37) derives from the azimuthal momentum equation (Eq. (4)). The term of the right-hand side is the magnetic tension. If $h_{\varpi}^{0} h_{\theta}^{0} \leq 0$, it decreases the rotation velocity (magnetic braking) and tends to increase it in the other case.

\subsubsection{Boundary conditions}

In this section, we address the problem of the boundary conditions.

- Thermal pressure

The cloud boundary $\left(\varpi_{b}, z_{b}\right)$ is given by the condition that the thermal pressure vanishes. Equations (13) lead to:

$P_{c}(\tau)-p_{\varpi} \varpi_{b}^{2}-p_{z} z_{b}^{2}=0$.

Thus, at the points $\left(a(\tau) l_{\varpi}(0), 0\right)$ and $\left(0, c(\tau) l_{z}(0)\right)$, we have with Eqs. (28)-(30):

$P^{0}=p_{\varpi}^{0}\left(\frac{\varpi_{b}}{a}\right)^{2}+p_{z}^{0}\left(\frac{z_{b}}{c}\right)^{2}=p_{\varpi}^{0} l_{\varpi}(0)^{2}=p_{z}^{0} l_{z}(0)^{2}$.

Therefore the boundary is a spheroid having axis lengths equal to: $l_{\varpi}(0)=\sqrt{P^{0} / p_{\varpi}^{0}}$ an $l_{z}(0)=\sqrt{P^{0} / p_{z}^{0}}$. We have consequently: $e(0)=\sqrt{1-p_{\varpi}^{0} / p_{z}^{0}}$ for an oblate cloud and $e(0)=\sqrt{1-p_{z}^{0} / p_{\varpi}^{0}}$ for a prolate cloud. In the case of a sphere, one has: $l_{\varpi}(0)=l_{z}(0)$ implying $p_{\varpi}^{0}=p_{z}^{0}$.

- Magnetic field

The component of the magnetic field which is perpendicular to the cloud surface must be continuous through the surface whereas the component which is tangent to the cloud surface can jump discontinuously from a value to another leading to surface electric currents.

Since the density of the external medium is zero, the Lorentz force must vanish in the external medium and the Alfvén speed is infinite.

This implies that the solutions given by Eqs. (35)-(37) with the condition stated by Eq. (49) are compatible with a poloidal magnetic field having the same structure (Eqs. (13)) in the external medium and in the cloud $\left(h_{\varpi} \neq 0\right.$ implies that the magnetic field becomes infinite when $r=\sqrt{\varpi^{2}+z^{2}} \rightarrow \infty$ ) and a vanishing toroidal magnetic component in the external medium. Note that the structure of the magnetic field does not necessarily follow the self-similar forms stated by Eqs. (13) in the external medium and that other structures may be solution of this problem.

The structure of the poloidal magnetic field in the external medium adjusts instantaneously as the boundary of the cloud moves, so that the field in the external medium remains forcefree, since the Alfvén speed is infinite.

\subsubsection{Energy balance}

In the hydrodynamical case $\left(h_{\theta}^{0}=h_{\varpi}^{0}=0\right)$, the solution is defined in a finite domain of space, and it is possible to find the energy of the corresponding cloud.

Let us multiply Eq. (35) by $2 l_{\varpi}(0)^{2} \dot{a}$, Eq. (36) by $l_{z}(0)^{2} \dot{c}$ and add the two expressions. Integrating the resulting equation, we obtain when $\gamma \neq 1$ :

$$
\begin{aligned}
& \frac{1}{2}\left(2 l_{\varpi}(0)^{2} \dot{a}^{2}+l_{z}(0)^{2} \dot{c}^{2}\right)+\frac{2}{\gamma-1} \frac{P^{0}}{a^{2 \gamma-2} c^{\gamma-1}} \\
& +\frac{l_{\varpi}(0)^{2} W^{2}(0)}{a^{2}}-\left(4 l_{\varpi}(0)^{2} \frac{A_{\varpi}}{c}+2 l_{z}(0)^{2} \frac{A_{z} c}{a^{2}}\right)=K .
\end{aligned}
$$

For $\gamma=1$, the integrated equation reads:

$$
\begin{aligned}
& \frac{1}{2}\left(2 l_{\varpi}(0)^{2} \dot{a}^{2}+l_{z}(0)^{2} \dot{c}^{2}\right)-2 P^{0} \ln \left(a^{2} c\right) \\
& \quad+\frac{l_{\varpi}(0)^{2} W^{2}(0)}{a^{2}}-\left(4 l_{\varpi}(0)^{2} \frac{A_{\varpi}}{c}+2 l_{z}(0)^{2} \frac{A_{z} c}{a^{2}}\right)=K,
\end{aligned}
$$

which is a first integral of Eqs. (35)-(36) and represents the total energy of the system. The first term is the kinetic energy, the second one is the thermal energy, the third one is the rotation energy and the last one is the gravitational energy.

If the magnetic field is non zero, the energy of the system is infinite since the magnetic energy of the surrounding gas is infinite and it is not possible to find such a relation.

The $\alpha$ and $\beta$ parameters, respectively the ratio of the thermal to gravitational energy and of the rotational to gravitational energy are equal to:

$\alpha(\tau)=\frac{2 P^{0}}{\gamma-1} \frac{a^{4-2 \gamma} c^{2-\gamma}}{\left(4 l_{\varpi}(0)^{2} A_{\varpi} a^{2}+2 l_{z}(0)^{2} A_{z} c^{2}\right)}$,

$\beta(\tau)=\frac{l_{\varpi}(0)^{2} W(0)^{2} c}{\left(4 l_{\varpi}(0)^{2} A_{\varpi} a^{2}+2 l_{z}(0)^{2} A_{z} c^{2}\right)}$.

In the case of an initially spherical cloud, we simply have: $\alpha(0)=2 P^{0} /(\gamma-1)$ and $\beta(0)=W(0)^{2}$.

\subsection{Potential and weaknesses of the approach}

Although the derivation of Eqs. (1)-(10) into Eqs. (35)-(37) is exact, the resulting reduction leaves aside some important physical effects. Nevertheless, it allows to catch some complex non-linear behaviours of the collapse of a rotating and magnetized polytropic cloud at very little cost. This approach complements a full numerical simulation for which all the parameter space cannot be easily explored and which can possibly be affected by the numerical resolution and by the numerical 
scheme. Finally such simple solutions can be used to derive semi-analytic criteria (see e.g. Tsuribe \& Inutsuka 1999).

We now discuss the potential and the weaknesses of this reduction.

\subsubsection{Potential}

Since the model is two dimensional, it is well suited to the study of the global behaviour of a collapsing core including the radial and axial rebounds. It allows to study the effects of rotation on the collapse, including the shape evolution and the subsequent variation of the gravitational potential.

Since the equation of state is polytropic, the model also allows to study the influence of the polytropic index on the cloud dynamics.

Since it includes magnetic field, it allows to catch some magnetic effects as well, like toroidal pinching and magnetic braking.

\subsubsection{Weaknesses}

The homologous reduction stated by Eqs. (13) excludes any complex hydrodynamical features like shocks and turbulence. Due to homology, the dissipation by viscosity vanishes.

The temperature decreases from the center to the cloud edge. This prevents the inwards propagation of the rarefaction wave that is usually obtained in the numerical simulations of cloud collapse.

The reduction stated by Eqs. (13) leads to a vanishing magnetic poloidal support. This clearly means that the solutions are not able to describe the cloud evolution if the poloidal magnetic support is the dominant process as it is the case for the collapse of a subcritical cloud controlled by ambipolar diffusion (e.g. Basu \& Mouschovias 1994).

In these solutions the growth (or the decrease) of the toroidal magnetic component due to the stretching of the poloidal component by differential (radial or axial) rotation vanishes, since the corresponding term cancels out in Eq. (24). This effect however is potentially important since the collapse could be induced by the toroidal magnetic pressure (Habe et al. 1991; Tomisaka 1991). The axial support due to the toroidal magnetic field vanishes as well.

\section{Isothermal cloud collapse: Phenomenology}

In this section we investigate the dynamical behaviour of the solutions of Eqs. (1)-(10) described by Eqs. (35)-(37) semianalytically. The collapse of a cold cloud (with no thermal pressure) has been studied by Lin et al. (1965). They find that the oblate clouds collapse into a disk whereas the prolate clouds collapse into a spindle. This is a purely gravitational effect. The gravitational force is higher along the minor axis than along the major one and the initial asymmetry is amplified.

\subsection{Application to dense cores}

As an application we will consider the collapse of an isothermal prestellar cloud. The density profiles of prestellar clouds have been found to be rather flat in the central part (e.g. Bacmann et al. 2000; Motte \& André 2001) having $\eta=\mathrm{d} \ln [\rho] / \mathrm{d} \ln [r]<$ 1. Consequently the uniform density approximation should be acceptable to describe the dynamical evolution of their inner part during the precollapse phase. Once the collapse phase begins, strong density gradients develop, the present approach is no more valid and other analytical techniques must be used to describe the collapse phase (see e.g. the references quoted in the introduction).

The typical physical parameters of the prestellar cloud are: temperature $\simeq 10 \mathrm{~K}$, density $\simeq 10^{4} \mathrm{~cm}^{-3}\left(\rho \simeq 3 \times 10^{-20} \mathrm{~g}\right)$, size $l_{0} \simeq 0.1 \mathrm{pc}$, adiabatic index $\gamma=1$, rotation ranging from $\beta=0$ to $\beta=0.1$, where $\beta$ is the ratio of the rotational energy to the gravitational energy. The magnetic field unit is $\sqrt{\mu_{0} \rho_{0}} \Omega_{J} l_{0} \simeq$ $25 \mu \mathrm{G}$. The time unit, $1 / \Omega_{J}$, is: $\simeq 2 \times 10^{5}$ years.

\subsubsection{Initial conditions}

As most of the observed cores are not spherical but have an observed aspect ratio of $\simeq 0.55$, we will consider initially oblate and prolate spheroids, starting with an initial aspect ratio around 0.5 .

For simplicity, we start with a thermal pressure such that the pressure force along the minor axis (along which it is stronger than along the major axis) is $3 / 4$ of the gravitational force along the same axis. The pressure along the major axis is then deduced from Eq. (49). We start with initial velocity equal to zero, $\dot{a}(0)=0$ and $\dot{c}(0)=0$.

\subsubsection{Numerical simulations}

In order to assess the validity of the solutions and understand in which regime they can be used, we perform hydrodynamical numerical simulations using the SPH code described in Hennebelle et al. (2003). We use 50000 particles to describe the cloud and a constant external pressure equal to the initial pressure of the cloud. In the following, we simulate the evolution of clouds of initially uniform density. Note that this configuration is not identical to the configuration described by Eqs. (35)-(37). In the simulation the rarefaction wave rapidly leads to non uniform density. Moreover the boundary pressure is not zero.

In order to have a realistic comparison, the mass of the cloud is chosen equal to one solar mass and the temperature to $10 \mathrm{~K}$.

\subsection{Oblate cloud}

We first consider non-rotating $(W(0)=0)$, unmagnetized $\left(q(0)=h_{\theta}^{0} / a(0)^{2} / c(0)=0\right)$, oblate clouds and then clouds with various rotation speeds.

\subsubsection{Non-rotating cloud}

We consider three different values of the aspect ratio, namely $r(0)=l_{z}(0) / l_{\varpi}(0)=0.7,0.5$ and 0.3 . The numerical integration is stopped when the density reaches the value $10^{5}$. 

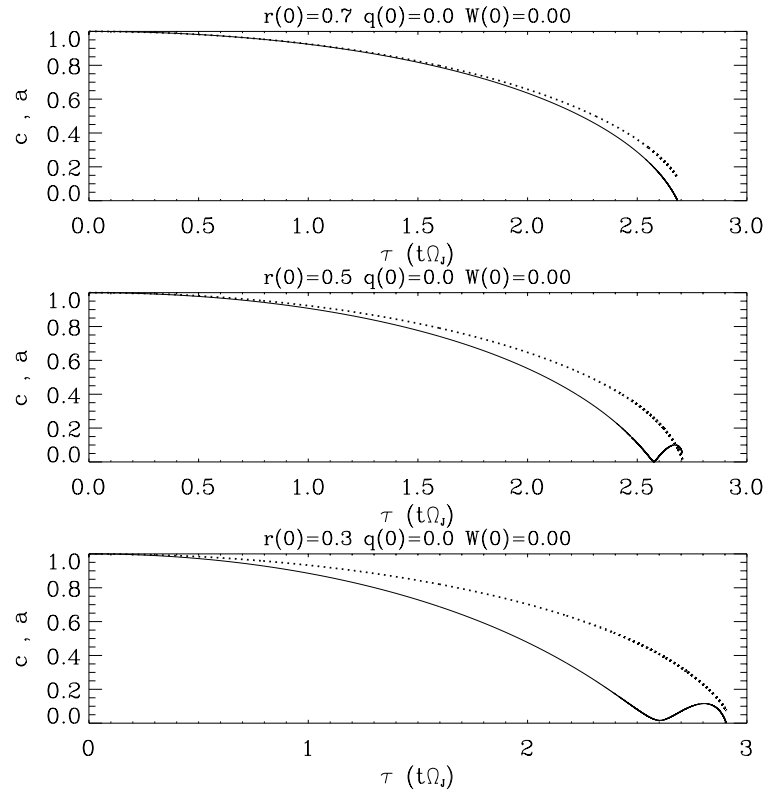

Fig. 1. Semi-analytical solutions of the collapse of an oblate isothermal $(\gamma=1)$ cloud with initial axis ratio equal to 0.7 (upper panel), 0.5 (middle panel) and 0.3 (lower panel). The evolution of the cloud radial axis contraction factor, $a(\tau)$ (dotted line), and the cloud polar axis contraction factor, $c(\tau)$ (full line), are displayed.

The results are displayed in Fig. 1, the evolution of $a(\tau)$, the radial axis (dotted line) and $c(\tau)$ the polar axis (full line) are presented.

- In the first case $(r(0)=0.7)$, it is seen that, as in the study of Lin et al. (1965) gravity amplifies the initial anisotropy and the cloud becomes more and more oblate. It eventually collapses into a disk at $t \simeq 2.7 / \Omega_{J}$.

- In the second case, the minor axis collapses more rapidly than the major axis but after a strong contraction $\left(c(0) / c_{\min } \simeq\right.$ $10^{3}$ ), a rebound due to thermal pressure occurs at $t \simeq 2.3 / \Omega_{J}$, the axial velocity becomes positive (outward motion) whereas the cloud still contracts radially. Let us recall that in their conclusion, Lin et al. (1965) predict that the cloud should oscillate if thermal pressure would be introduced. The cloud finally collapses into a spindle at $t=2.6 / \Omega_{J}$. Shortly before, the collapse along the polar axis restarts. Note that the rebound is very stiff and some care is required in the numerical resolution. We use adaptive time steps imposing that the maximum variation of $a$ and $c$ between two steps is less than $1 \%$ and we checked that the same result is obtained by requiring a variation of $0.5 \%$. We also check that the energy given by Eq. (51) is well conserved.

- In the third case $(r(0)=0.3)$, the rebound occurs still at $t \simeq 2.6 / \Omega_{J}$ but the contraction is weaker $\left(c(0) / c_{\min } \simeq 1 / 30\right)$. This is due to the fact that since the cloud is initially more oblate than in the previous case, the axial velocity is smaller leading to a weaker contraction. Also the dynamical time scale along the minor axis is shorter and the radial contraction is weaker when the rebound occurs and the density is smaller by the time of this rebound. After this rebound, the collapse along the polar axis restarts and finally, the cloud collapses into a disk, i.e. the density reaches our threshold, at $t \simeq 2.9 / \Omega_{J}$.
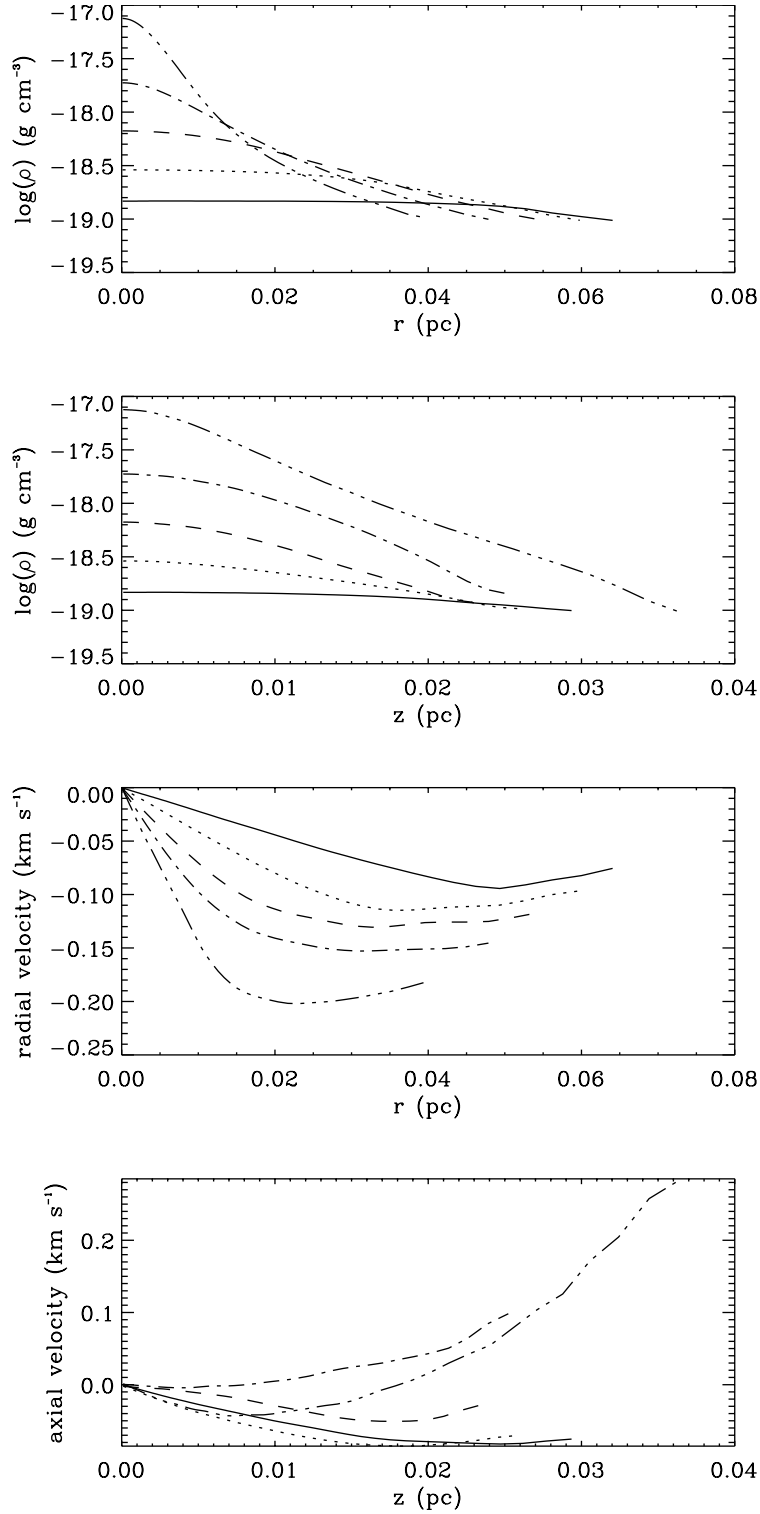

Fig. 2. Numerical simulation of a rotating oblate and isothermal $(\gamma=$ 1) cloud with initial axis ratio equal to 0.5 . First panel displays the equatorial density, second panel the density along the polar axis, third is the radial velocity at the equator and fourth is the axial velocity along the polar axis. Full line corresponds to $t=0.89$, dotted line to 1.34 , dashed to 1.78 , dot-dashed to 2.23 and triple-dot-dashed to $2.67 / \Omega_{J}$.

- Figure 2 presents the numerical simulation of a non rotating oblate cloud (the initial aspect ratio equal is 0.5 ). The initial length of the major axis is $0.07 \mathrm{pc}$. Five time steps are displayed. It is seen that the velocity field (both radial and axial components) can be well approximated by an homologous law in the inner part and that the inner part is also reasonably flat during the precollapse phase. Because of the rarefaction wave, not describe by the semi-analytical solutions, the density contrast in the outer part increases and the velocity is no more homologous. It is seen that at time $t=2.23 / \Omega_{J}$ the cloud bounces axially because of the thermal pressure. Note that the inner part slows down axially and stops collapsing $(t=1.78$ and $2.23 / \Omega_{J}$ ) without bouncing. The evolution of the radial 

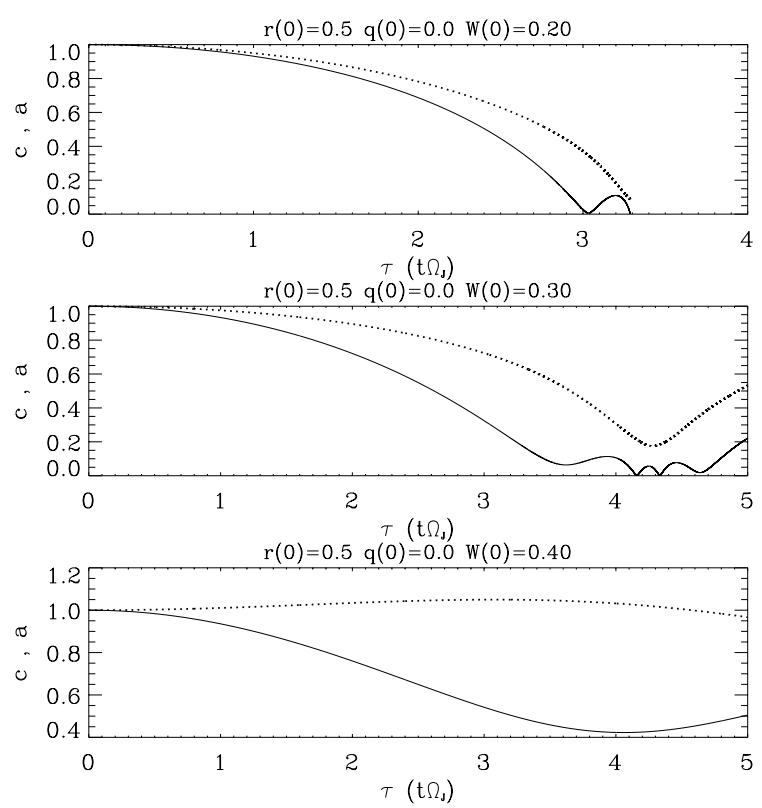

Fig. 3. Semi-analytical solutions of the collapse of a rotating oblate and isothermal $(\gamma=1)$ cloud with initial axis ratio equal to 0.5 . Upper panel is $W(0)=0.2$, middle one, $W(0)=0.3$ and lower panel is $W(0)=0.4$. The evolution of $a(\tau)$ (dotted line) and $c(\tau)$ (full line) are displayed.

component, $V_{\varpi}$ is monotonous in the inner and in the outer part. At time $2.67 / \Omega_{J}$ it is seen that the collapse along the polar axis has restarted in the inner part whereas the outer part is still bouncing. Although this behaviour is too complex to be completely described by the semi-analytical solutions, they nevertheless undoubtedly reproduce some complex features of the cloud evolution including the axial rebounds and subsequent collapse. Note that the largest density reached before the rebound is significantly overestimated by the semi-analytical solution. This is due to the thermal pressure which is not accurately calculated. However, the duration of this phase of strong condensation is short and does not alter much the subsequent evolution.

\subsubsection{Rotating oblate cloud}

We consider the evolution of an initially oblate cloud with initial axis ratio $r(0)=0.5$ and investigate three initial rotation values, $W(0)=0.2,0.3$ and 0.4 . The results are displayed in Fig. 3.

- The first case $(W(0)=0.2)$ is similar to the evolution of the non-rotating clouds (see Fig. 1), except that due to the centrifugal support, the rebound occurs later $\left(t \simeq 3 / \Omega_{J}\right)$. The cloud collapses into a disk at $t \simeq 3.3 / \Omega_{J}$.

- In the second case $(W(0)=0.3)$, it is found that the cloud does not collapse but makes a series of rebounds. This is due to the fact that rotation strongly supports the cloud and consequently the contraction along the polar axis is too weak (density is lower and gravitational force along the polar axis smaller than for the previous case) for allowing the density to reach our threshold. The cloud presents a quasi-periodic behaviour. Since Eqs. (35)-(36) are non-dissipative, the cloud never relax
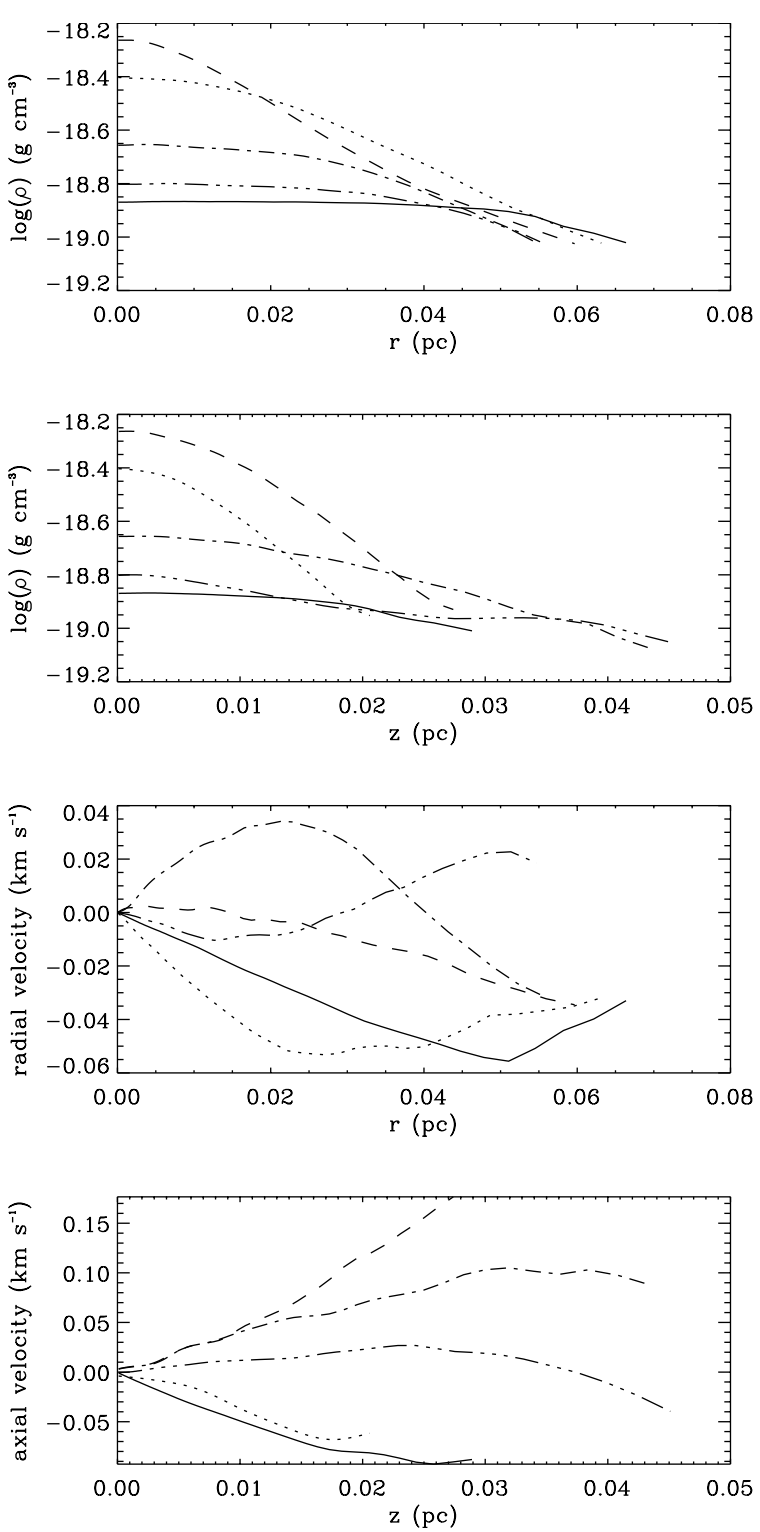

Fig. 4. Same as Fig. 2 for a rotating oblate cloud (initial aspect ratio equal to 0.5 and $W(0)=0.3$ ). Full line corresponds to $t=0.89$, dotted line to 1.78 , dashed to 2.68 , dot-dashed to 3.57 and triple-dot-dashed to $t=4.46 / \Omega_{J}$.

and never collapse. In a more realistic situation, the number of rebounds depend on the typical time scale of the dissipative processes. If this time is short compared to the oscillation period, the rebounds will be damped rapidly (see Fig. 5 for a numerical estimate of this time).

- In the third case $(W(0)=0.4)$, the centrifugal force is stronger than for the previous case and the oscillations have a longer period and a smaller amplitude.

- Figure 4 presents the numerical simulation of a rotating $(W(0)=0.3)$ initially oblate core $(r(0)=0.5)$. The initial length of the major axis is $0.07 \mathrm{pc}$. As in the case studied in the previous section, it is seen that the homologous approximation is fair to describe the velocity field and that the cloud is rather flat in the inner part. The cloud starts to contract axially and radially. At time $t=2.68 / \Omega_{J}$ the cloud rebounds axially and at time $3.57 / \Omega_{J}$, the inner part rebounds radially whereas the 


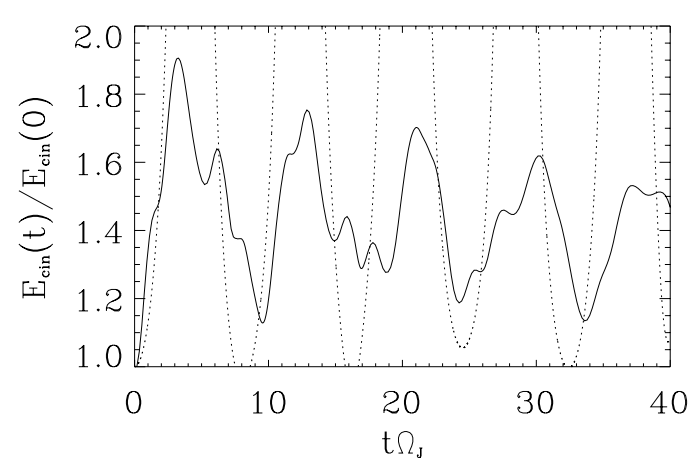

Fig. 5. The kinetical energy as a function of time. Full line is the numerical solution and dotted line is the semi-analytical one.

outer part is still contracting. At time $t=4.46 / \Omega_{J}$ the outer part of the cloud expands radially whereas the inner part is contracting. This behaviour is again very similar (though more complex) to the case displayed in the second panel of Fig. 3 where the axial rebound occurs at time $\simeq 3.5 / \Omega_{J}$ and the radial one at $\simeq 4.2 / \Omega_{J}$. The disagreement on the times is about $20 \%$.

During the subsequent evolution, the cloud continue to bounce axially and radially several times and looses its energy slowly as can be seen in Fig. 5 where the evolution of the kinematical energy of the cloud is displayed. The minima correspond to the most expanded states and the maxima are close to the most condensed. It is seen that the global behaviour of the cloud is reproduced by the semi-analytical solution during few oscillation cycles. The details however are not accurately described. In particular, as already mentioned, the semi-analytical solutions overestimate the largest density reached during the oscillation cycles.

It is worth noting that such an oscillating behaviour may have been recently observed in the dark cloud Barnard 68 by Lada et al. (2003) where both systematic inwards and outward motions have been inferred.

\subsection{Prolate cloud}

We consider now the collapse of prolate clouds, i.e. clouds having filamentary shape with an initial aspect ratio $r(0)=$ $l_{\varpi}(0) / l_{z}(0)=0.5$. We first investigate the case of rotating and unmagnetized clouds and then rotating and magnetized filaments.

\subsubsection{Rotating unmagnetized cloud}

Three values of the rotation speed are considered, $W(0)=$ 0, 0.15 and 0.3. The results are displayed in Fig. 6 .

- The first case (upper panel) is similar to the result of Lin et al. (1965). The major axis collapses first since gravity is stronger in this direction than along the major axis $\left(A_{z} / A_{\varpi} \simeq\right.$ 0.42 ) and the cloud collapses into a spindle.

- In the second case $(W(0)=0.15)$, it is seen that the collapse of the minor axis is delayed by the centrifugal support and that the dynamics of the two axis is comparable.

- When the cloud rotates more rapidly $(W(0)=0.3)$, the centrifugal force is stronger and the cloud collapses more
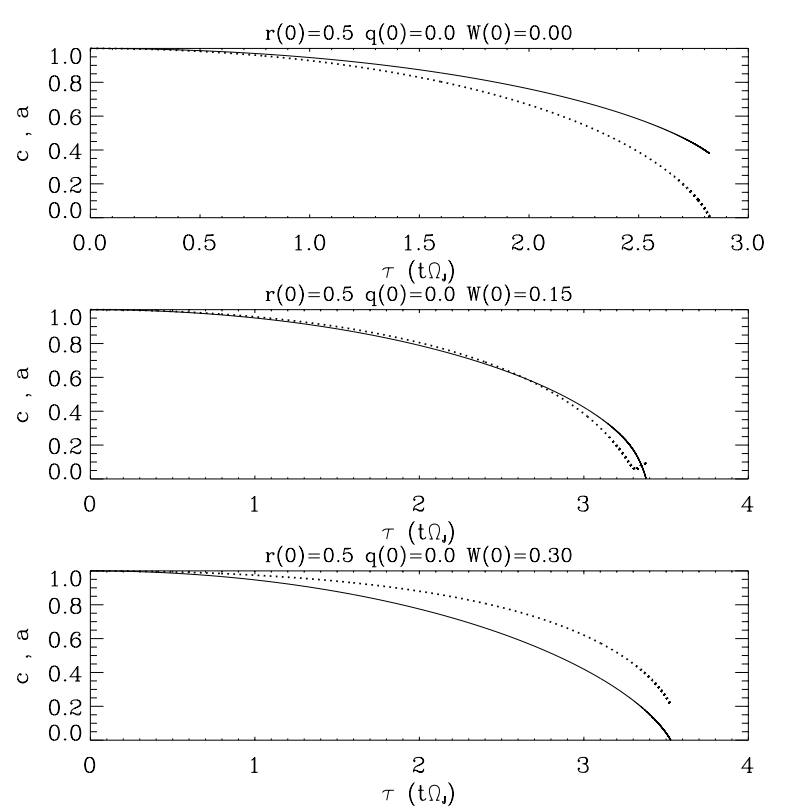

Fig. 6. Semi-analytical solutions of the collapse of a rotating prolate isothermal $(\gamma=1)$ cloud with initial axis ratio equal to $r(0)=0.5$. Upper panel is $W(0)=0$ (no rotation), middle panel, $W(0)=0.15$ and lower panel, $W(0)=0.3$. The evolution of $a(\tau)$ (dotted line) and $c(\tau)$ (full line) are displayed.

slowly in the radial direction than along the polar axis. The cloud collapses into a disk at time $t \simeq 3.5 / \Omega_{J}$.

\subsubsection{Rotating and magnetized cloud}

We investigate now the collapse of a rotating and magnetized cloud. For simplicity, we consider the same initial condition as in Sect. 3.3.1 and add a toroidal magnetic field with an amplitude $q=0.3$ and 0.7. The results are displayed in Fig. 7 .

- In the first case $(W(0)=0.1, q(0)=0.3)$, the evolution is similar to the non rotating prolate cloud $(W(0)=0, q(0)=0)$ but the minor axis collapses more rapidly. This is due to the toroidal magnetic field that adds up to gravity in the equatorial plane but not along the polar axis (initially we have, $A_{z} /\left(A_{\varpi}+\right.$ $\left.q(0)^{2}\right) \simeq 0.29$ ). At time $t=1.9 / \Omega_{J}$ a radial rebound occurs because rotation prevents the collapse. The cloud then becomes less and less prolate, until $t \simeq 2.5 / \Omega_{J}$, where the collapse in the radial direction restarts. It eventually collapses into a disk at time $t \simeq 2.7 / \Omega_{J}$.

- If the toroidal magnetic field is stronger $(q(0)=0.7)$, the cloud is more strongly squeezed $\left(A_{z} /\left(A_{\varpi}+q(0)^{2}\right) \simeq 0.12\right)$ and the minor axis collapses more rapidly than in the previous case and at $t \simeq 1.2 / \Omega_{J}$, the first rebound occurs. By this time, the major axis has little evolved because its dynamical time scale is longer. At $t \simeq 1.9 / \Omega_{J}$, the collapse in the radial direction restarts and the cloud makes a second rebound at time $t \simeq 2.4 / \Omega_{J}$. It eventually collapses into a disk at $t \simeq 2.5 / \Omega_{J}$.

- The evolution of the more rapidly rotating cloud $(W(0)=$ 0.3 ) is very similar to the slowly rotating one. The only difference appears when rotation becomes significant, i.e. during the contractions and the subsequent rebounds at $t \simeq 1.2$ and $2.5 / \Omega_{J}$. This is due to the fact that in these two cases 

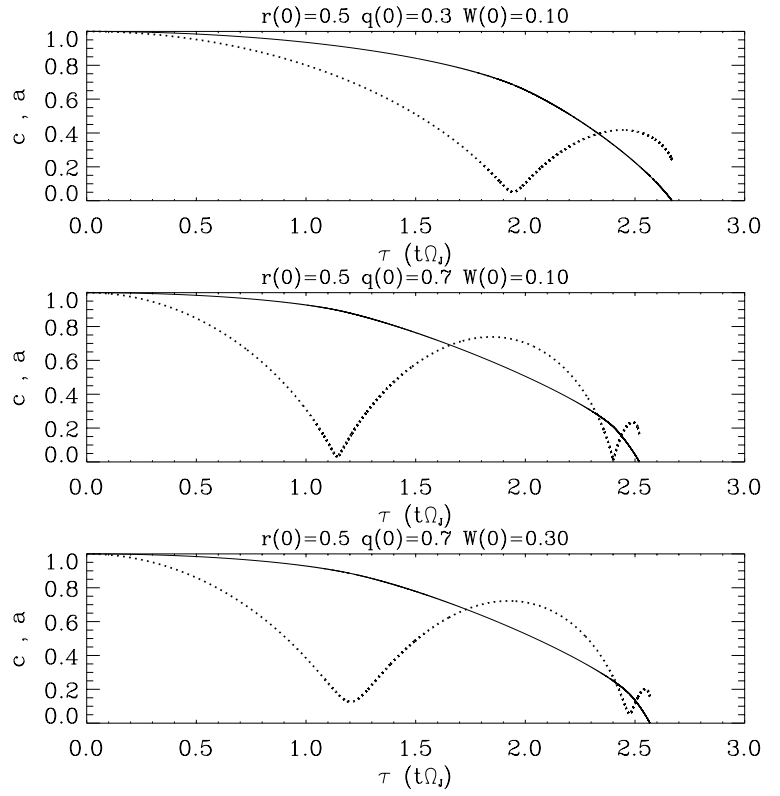

Fig. 7. Semi-analytical solutions of the collapse of a rotating and magnetized prolate isothermal $(\gamma=1)$ cloud with initial axis ratio equal to $r(0)=0.5$. Upper panel is $W(0)=0.1, q(0)=0.3$ middle panel, $W(0)=0.1, q(0)=0.7$ and lower panel, $W(0)=0.3, q(0)=0.7$. The evolution of $a(\tau)$ (dotted line) and $c(\tau)$ (full line) are displayed.

the centrifugal force is small compared to the toroidal pinching when the cloud is weakly condensed. The centrifugal force plays an important rôle only when the cloud is significantly radially condensed.

Let us summarise our conclusions. The collapse of a prolate and magnetized core (with strong toroidal pinching) occurs slowly through gravity along the polar axis and rapidly through toroidal pinching and gravity in the radial direction. Since rotation prevents radial collapse, few rebounds occur during the slow collapse along the polar axis and finally the cloud collapses into a disk. Because of the toroidal magnetic field, the dynamical time scale of the minor axis is much shorter than the dynamical time scale of the major one. This process depends weakly on the rotation amplitude (as long as it is neither zero nor very large) and on the thermal pressure.

\section{Apparent shape of the clouds, comparison with observation}

In spite of the important restrictions listed in Sect. 2.5.2, the solutions presented in this paper enable the study of the cloud shape evolution during the precollapse phase. It is worth trying to compare the derived predictions with observations of dense cores traced by the $\mathrm{NH}_{3}$ molecule (Jijina et al. 1999; Lee \& Myers 1999). Since our simple model assumes a uniform density and does not include complex processes like accretion or protostellar outflows, we select the starless dense cores only from the Jijina et al. (1999) catalog.

Two recent studies (Jones et al. 2001; Goodwin et al. 2001) analyse the observed apparent axis ratio distribution derived from this catalog and conclude that this distribution is compatible with dense cores being triaxal.
Since our model makes restrictive assumptions and since the Jijina et al. (1999) catalog is not an homogeneous sample, the cores having been observed with various instruments and at various resolutions, the present analysis is rather tentative. The idea is to see if a good fit can be obtained and for which range of parameters. The result will not be more than indicative.

\subsection{Method}

The first step of our analysis is to derive a shape distribution from the time sequence of $a$ and $c$. This is achieved by counting the fraction of time that one cloud spends with a given axis ratio.

The second step is to project the axis ratio distribution along the line of sight. Let $\psi$ be the axis ratio distribution, and $\phi$, the apparent (or observed) axis ratio distribution. The projection along the line of sight is achieved according to the law (Binney 1978; Fall \& Freck 1983):

$\phi(r)=r \int_{0}^{r} \mathrm{~d} s\left(1-s^{2}\right)^{-1 / 2}\left(r^{2}-s^{2}\right)^{-1 / 2} \psi(s)$

for an oblate distribution,

$\phi(r)=r^{-2} \int_{0}^{r} \mathrm{~d} s s^{2}\left(1-s^{2}\right)^{-1 / 2}\left(r^{2}-s^{2}\right)^{-1 / 2} \psi(s)$

for a prolate distribution.

The third step is to calculate the $\chi^{2}$ between this theoretical distribution and the distribution derived from the catalog of Jinina et al. (1999) which includes 79 starless cores.

We explore a large set of parameters:

- the ratio between the 2 axis, $r(0)=l_{z}(0) / l_{\varpi}(0)$ varies from 0.5 (oblate cloud) to 2.2 (prolate cloud), with a regular sampling of 0.1 leading to 18 values;

- the magnetic toroidal intensity $q(0)$ varies from 0 to 1.5 (16 values), in physical units it ranges from 0 to $40 \mu \mathrm{G}$;

- the rotation velocity, $W(0)$, from 0 to 0.6 (7 values) which corresponds to $\beta$ ranging from 0 to $36 \%$;

- the thermal pressure from $p_{i}^{0}=\left(A_{i}(0)\right) / 10$ to $A_{i}(0)$ (10 values) where $i=z$ for oblate cores and $i=\varpi$ for prolate cores.

Consequently, we solve $18 \times 16 \times 7 \times 10\left(\simeq 2 \times 10^{4}\right)$ times Eqs. (35)-(36) and calculate the corresponding apparent axis ratio and the $\chi^{2}$ with the observed distribution for each of these solutions.

\subsection{Result}

The best agreement obtained is for $l_{\varpi}(0) / l_{z}(0)=0.5, q=0.7$, $W(0)=0.3, P_{c}=A_{\varpi}(0) / 7$, which is very close to the second and third cases we have considered in Sect. 3.3.2 (prolate magnetized cores). The theoretical (full line) and observational (dot-dashed line) are displayed in the third panel of Fig. 8. The agreement is good and better than in the study of Jones et al. (2001) and Goodwin et al. (2002). The corresponding value of $\chi^{2}$ is: 0.56 (assuming an observational Poisson noise and no theoretical noise). The distribution depends weakly on the 

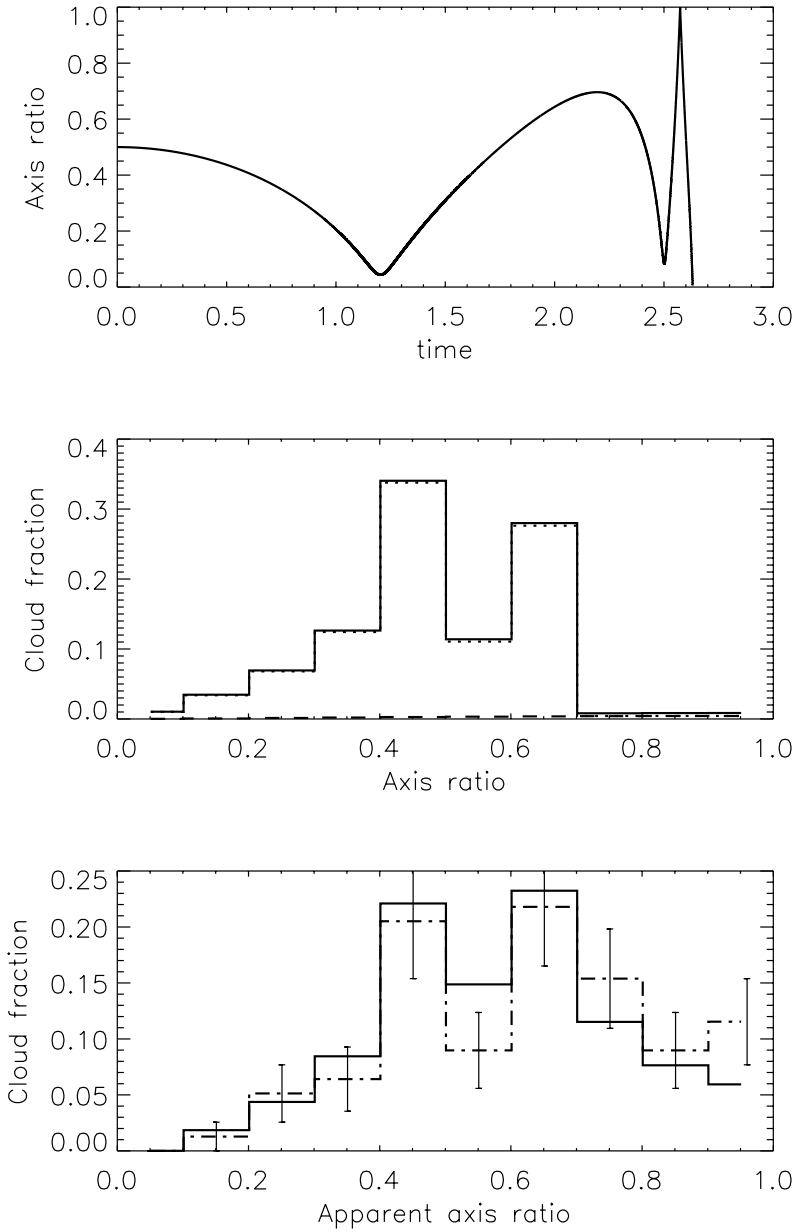

Fig. 8. Apparent axis ratio during the cloud evolution (upper panel), axis ratio distribution (middle panel) and apparent axis ratio distribution (lower panel). The dotted line (second panel) is the fraction of prolate core, the dashed line, the fraction of oblate cores. In the third panel the dot-dashed curve is the apparent axis distribution of starless cores belonging to the catalog of Jijina et al. (1999) whereas the full line is the theoretical distribution obtained for the initial conditions $l_{\varpi}(0) / l_{z}(0)=0.5, q=0.7$ (magnetic toroidal intensity), $W(0)=0.3$ (rotation) and $p_{\varpi}^{0}=A_{\varpi}(0) / 7$ (thermal pressure). The value of $\chi^{2}$ is 0.56 .

value of rotation and thermal pressure and is much sensitive to the initial axis ratio $r(0)=l_{\varpi}(0) / l_{z}(0)$ and to the magnetic toroidal intensity.

We find 179 cases with $\chi<1$ (87 have $\chi<0.8)$ similar to the best case. They have $l_{z}(0) / l_{\varpi}(0)$ equal to 2 to $2.2, q$ equal to 0.6 to 0.9 and all possible values of $W(0)$ and $P_{c}$.

We also find 59 cases that belong to another family. They are oblate $\left(l_{z}(0) / l_{\varpi}(0)=0.5\right)$ fast rotating cores $(W(0) \geq 0.4)$ similar to the cases presented in Sect. 3.2.2. However, the smallest $\chi$ is 0.85 and most of them have $\chi>0.9$. The most important disagreement is due to the fact that too many clouds with apparent axis ratio close to 1 , are found.

\subsection{Discussion}

The apparent axis ratio distribution derived from the evolution of the prolate magnetized filaments agrees with the observed distribution.

The rotating oblate cores agree less well with the observational apparent axis ratio distribution. Indeed the core remains oblate and Ryden (1996) shows that an oblate core distribution cannot explained the observed apparent axis ratio distribution.

We would like to emphasize the fact that the axis ratio distribution depends weakly on the initial rotation and thermal pressure values (almost all values of these parameters are compatible with the observed axis ratio distribution) and strongly on the initial axis ratio and on the toroidal magnetic field. Consequently, the agreement is obtained by adjusting two parameters only whereas there are 9 bins in the fitted distribution. Therefore the fact that we obtain a parameter range that fits the data is not a trivial consequence of a large number of parameters. Indeed there would not be acceptable fits without a toroidal magnetic field.

The most striking feature of the unprojected axis ratio distribution of the example displayed in Fig. 8 is that it is bimodal (with two peaks around $p_{1} \simeq 0.5$ and $p_{2} \simeq 0.7$ ). This is due to the fact that the cloud spends a long time, with its initial aspect ratio and a long time with the aspect ratio it has after the first rebound. This feature is smoothed by the projection effect and the apparent axis ratio distribution is only slightly or marginally bimodal.

The data of Jijina et al. (1999) are compatible with (though marginally statistically significant) a starless cores apparent axis ratio distribution being bimodal (see the dot-dashed curve of Fig. 8 or the starless cores axis ratio distribution showed in Goodwin et al. 2002). The same trend appears also in two of the subsamples studied by Ryden (1996) and in the data studied recently by Curry (2002) although in all of these samples the poor statistic precludes a definite answer. More data are needed to confirm or to reject this feature.

\section{Conclusion}

New solutions of the gravo-magnetic contraction have been presented. They describe the evolution of a uniform density cloud with spheroidal shape and can be applied in various situations. These solutions allow to explore a wide set of parameters and constitute a useful complement to numerical simulations. They can also be used as benchmarks in problems involving gravitational collapse.

We have explored the phenomenology of the gravitational contraction of isothermal dense cores described by these semianalytical solutions with special emphasis on the axial and radial rebounds. Numerical simulations have been performed to compare these solutions with the evolution of an isothermal initially uniform cloud and it has been found that they can reproduce some aspects of cloud evolution during the precollapse phase. The most important disagreement is the cloud outer part which is significantly influenced by the rarefaction wave not taken into account in the semi-analytical solutions. 
For each of a large set of initial values, we have calculated the apparent axis ratio distribution resulting from the evolution of the core and tentatively, compared it to the observational one derived from the catalog of Jijina et al. (1999). We find that the axis ratio distribution of a collapsing rotating, prolate (initial aspect ratio $\simeq 0.5)$ magnetized $(\simeq 17-20 \mu \mathrm{G}$ for a density of $\simeq 10^{4} \mathrm{~cm}^{-3}$ ) filament is in good agreement with the observational distribution (see Fig. 8). Definite conclusion cannot be drawn at this stage however since our model is very simplified and (mhd) numerical studies are required to confirm the results. Also the observational core sample is not homogeneous and the statistical significance remains poor. Finally alternative distributions of evolving or static cores can fit the observational data as well. Nevertheless, we believe that prolate cores permeated by an helical magnetic field deserve further investigations.

Acknowledgements. I thank Philippe André, Simon Goodwin, Derek Ward-Thompson and Anthony Whitworth for stimulating discussions. I acknowledge a critical reading of the manuscript by Michel Pérault. I thank Jason Fiege, the referee for very insightful and detailed reports. I gratefully acknowledge the support of an European Commission Research Training Network under the Fifth Framework Programme (No. HPRN-CT2000-00155) and a CNES fellowship.

\section{References}

Aburihan, M., Fiege, J., Henriksen, R., \& Levy, T. 2001, MNRAS, 326, 1217

Bacmann, A., André, P., \& Puget, J.-L. 2000, A\&A, 361, 555

Basu, S., \& Mouschovias, T. 1994, ApJ, 432, 720

Binney, J. 1978, MNRAS, 183, 501

Binney, J., \& Tremaine, S. 1987, Galactic dynamics (Princeton: Princeton Univ. Press)

Bouquet, S., Feix, M., \& Fijalkow, E. 1985, ApJ, 293, 494

Chandrasekhar, S. 1969, Ellipsoidal Figures of Equilibrium (New Haven: Yale University Press)

Curry, C. 2000, ApJ, 541, 831
Curry, C. 2002, ApJ, 576, 914

Curry, C., \& Stahler, S. 2001, ApJ, 555, 160

Fall, S., \& Frenk, C. 1983, AJ, 88, 1626

Ferrara, A., \& Shchekinov, Y. 1993, Geophys. Astrophys. Fluid Dynamics, 84, 274

Fiege, J., \& Pudritz, R. 2000a, MNRAS, 311, 85

Fiege, J., \& Pudritz, R. 2000b, MNRAS, 311, 105

Fiege, J., \& Pudritz, R. 2000c, ApJ, 534, 291

Foster, P. N. \& Chevalier, R. A. 1993, ApJ, 416, 303

Goodwin, S., Ward-Thompson, D., \& Whitworth, A. 2002, MNRAS, 330, 769

Habe, A., Uchida, Y., Ikeuchi, S., \& Pudritz, R. 1991, PASJ, 43, 703

Hartmann, L. 2002, ApJ, 578, 914

Hennebelle, P. 2001, A\&A, 378, 214

Hennebelle, P. 2003, A\&A, 397, 381

Hennebelle, P., \& Pérault, M. 2000, A\&A, 359, 1124

Hennebelle, P., Whithworth, A., Gladwin, P., \& André, P. 2003, MNRAS, 340, 870

Hunter, C. 1977, ApJ, 218, 834

Jijina, J., Myers, P., \& Adams, F. 1999, ApJS, 125, 161

Jones, C., Basu, S., \& Dubinski, J. 2001, ApJ, 551, 387

Lada, C., Bergin, E., Alves, J., \& Huard, T. 2003, ApJ, 586, L286

Larson, R. 1969, MNRAS, 145, 271

Lin, C., Mestel, L., \& Shu, F. 1965, ApJ, 142, 1431

Mestel, L. 1963, MNRAS, 126, 553

Motte, F., \& André, P. 2001, A\&A, 365, 440

Mouschovias, T. 1976, ApJ, 207, 141

Myers, P., Fuller, G., Goodman, A., \& Benson, P. 1991, ApJ, 376, 551

Nakamura, F., Hanawa, T., \& Nakano, T. 1993, PASJ, 45, 551

Olver, P. 1986, Applications of Lie-groups to differential Equations (New-York: Springer-Verlag)

Penston, M. 1969a, MNRAS, 144, 425

Penston, M. 1969b, MNRAS, 145, 457

Ryden, B. 1996, ApJ, 471, 822

Shu, F. 1977, ApJ, 214, 488

Tomisaka, K., Ikeuchi, S., \& Nakamura, T. 1988, ApJ, 326, 208

Tomisaka, K. 1991, ApJ, 376, 190

Tsuribe, T., \& Inutsuka, S. 1999, ApJ, 526, 307

Whitworth, A., \& Summers, D. 1985, MNRAS, 214, 1 